\title{
Esterco bovino e calagem para formação de mudas de guanandi
}

\author{
Adriana Guirado Artur ${ }^{(1)}$, Mara Cristina Pessôa da Cruz $^{(1)}$, Manoel Evaristo Ferreira(1), \\ Vitor Corrêa de Mattos Barretto ${ }^{(2)}$ e Renato Yagi(1)
}

\begin{abstract}
(1)Universidade Estadual Paulista (Unesp), Fac. de Ciências Agrárias e Veterinárias (Fcav), Dep. de Solos e Adubos, Via de Acesso Prof. Paulo Donato Castellane, s/no, CEP 14884-900 Jaboticabal, SP. E-mail: driguirado@yahoo.com.br, mcpcruz@fcav.unesp.br, evaristo@fcav.unesp.br, renatoyagi@yahoo.com.br(2)Unesp, Fcav, Dep. de Produção Vegetal. E-mail: barretto@fcav.unesp.br
\end{abstract}

\begin{abstract}
Resumo - O objetivo deste trabalho foi avaliar, em casa de vegetação, os efeitos de doses de esterco bovino e de calcário na formação de mudas de guanandi (Calophyllum brasiliense Cambèss.). Foram avaliadas quatro doses de cada fator, combinadas em esquema fatorial $4 \times 4$. $\mathrm{O}$ delineamento experimental foi inteiramente ao acaso com quatro repetições, e cada parcela continha cinco mudas. O substrato foi formado pela mistura, em volume, de oito partes de subsolo e duas partes de areia, e doses de esterco correspondentes a 0, 2, 4 e 6 partes que, em massa, equivalem a $0,101,175$ e $229 \mathrm{~kg} \mathrm{~m}^{-3}$. As doses de calcário foram calculadas para elevar a saturação por bases (V\%) a 20,35, 50 e 65\%. Aos 120 dias, foram avaliados: altura, diâmetro do colo, número de folhas, área foliar e matéria seca das partes aérea e radicular das mudas. Em substrato constituído de subsolo+areia, a calagem e a adubação com esterco bovino não são necessárias para produção de mudas de guanandi.
\end{abstract}

Termos para indexação: Calophyllum brasiliense, adubação orgânica, substrato.

\section{Cattle manure and liming for guanandi seedlings production}

\begin{abstract}
Abstrat - The effects of cattle manure and lime levels in substrate were evaluated to produce guanandi (Calophyllum brasiliense Cambèss.) seedlings in greenhouse conditions. Four levels of each factor were evaluated, in a factorial scheme $4 \times 4$. The experimental design was completely randomized with four replicates, each plot with five seedlings. The substrate was obtained by the mixture of eight subsoil parts and two sand parts (v:v). The manure was applied in 0,2, 4 and 6 parts, in volume, that correspond, in mass, to $0,101,175$ and $229 \mathrm{~kg} \mathrm{~m}^{-3}$. Lime doses were calculated to increase the base saturation degree near to $20 \%$ (original subsoil sample), 35, 50 and $65 \%$. Seedlings harvest was performed 120 days after transplant, when height, stem diameter, leaf number, leaf area, shoot and root dry matter were evaluated. For substrate constituted by subsoil+sand, the liming and the cattle manure are not necessary to produce guanandi seedlings.
\end{abstract}

Index terms: Calophyllum brasiliense, organic fertilizer, substrate.

\section{Introdução}

Nos últimos anos, a procura por mudas de espécies florestais nativas tem crescido no Brasil em razão da necessidade de reflorestamento. Nos projetos de revegetação de áreas degradadas, tem sido explorado o potencial das espécies nativas regionais, supostamente melhor adaptadas às condições edafoclimáticas, o que facilita o restabelecimento do equilíbrio entre a fauna e a flora (Fernandes et al., 2000).

$\mathrm{Na}$ formação das mudas, a escolha do substrato deve ser feita de acordo com a disponibilidade de materiais, e o subsolo é um dos componentes mais utilizados no preenchimento das embalagens de plástico. O substrato deve ser preferencialmente argilo-arenoso para que, ao ser retirado do saco de plástico, por ocasião do plantio, o torrão com a muda não se quebre facilmente e ocasione perda de mudas no campo (Gomes \& Couto, 1986).

Quanto aos atributos químicos, o subsolo geralmente é mais ácido e contém teores menores de nutrientes, mas pode ser facilmente corrigido com aplicação de calcário e de adubos minerais e orgânicos. A correção da acidez do substrato pode favorecer a produção de mudas, como constatado por Furtini Neto et al. (1999) em cássia-verrugosa, ipê-mirim, angico-do-cerrado e cedro. No entanto, conforme a espécie e os atributos químicos do subsolo, a resposta à calagem pode não ocorrer, como relatado por Balieiro et al. (2001) em duas espécies de acácias. 
Os adubos orgânicos são as fontes de nutrientes de uso mais freqüente na composição de substratos, têm atuação relevante na melhoria dos seus atributos físicos e estimulam os processos microbianos. Entre os adubos orgânicos, o esterco bovino é o mais usado e tem levado a bons resultados na produção de mudas de espécies florestais (Castro et al., 1996; Tedesco et al., 1999; Carvalho Filho et al., 2004).

O guanandi (Calophyllum brasiliense Cambèss.), espécie considerada climácica por apresentar regeneração abundante na sombra, tem crescido em importância pelo fato de sua madeira ser resistente à água, ter boa durabilidade e ter sido citada por especialistas como substituta do mogno (Carvalho, 1994). O crescimento da planta é monopodial, característica que proporciona fustes bem definidos. Pode chegar a $40 \mathrm{~m}$ de altura e $1,5 \mathrm{~m}$ de diâmetro e o ponto de corte adequado é atingido com cerca de 18,5 anos (Carvalho, 1994).

A maioria das informações sobre o guanandi e muitas outras espécies florestais relaciona-se principalmente às características botânicas e dendrológicas, mas informações sobre exigências nutricionais são escassas (Carvalho, 1994).

O objetivo deste trabalho foi avaliar os efeitos de doses de esterco bovino associadas a doses de calcário na formação de mudas de guanandi.

\section{Material e Métodos}

Um experimento foi conduzido em casa de vegetação no período de 23 de maio a 23 de setembro de 2006, na Faculdade de Ciências Agrárias e Veterinárias, Campus da Unesp de Jaboticabal, a fim de avaliar os efeitos de doses de esterco bovino associadas a doses de calcário na formação de mudas de guanandi.

Foi utilizada amostra de subsolo de um Latossolo Vermelho de textura argilosa, que foi secada ao ar, passada em peneira de $4 \mathrm{~mm}$ e homogeneizada. Os atributos químicos do subsolo, determinados de acordo com os métodos descritos em Raij et al. (2001), são os seguintes: $\mathrm{P}$ resina, $4 \mathrm{mg} \mathrm{dm}^{-3} ; \mathrm{MO}, 9 \mathrm{~g} \mathrm{dm}^{-3} ; \mathrm{pH}$ em $\mathrm{CaCl}_{2}, 4,9 ; \mathrm{K}^{+}, 0,3 \mathrm{mmol}_{\mathrm{c}} \mathrm{dm}^{-3} ; \mathrm{Ca}^{2+}, 5 \mathrm{mmol}_{\mathrm{c}} \mathrm{dm}^{-3}$; $\mathrm{Mg}^{2+}, 2 \mathrm{mmol}_{\mathrm{c}} \mathrm{dm}^{-3} ; \mathrm{Al}^{3+}, 6 \mathrm{mmol}_{\mathrm{c}} \mathrm{dm}^{-3} ; \mathrm{H}+\mathrm{Al}$, $28 \mathrm{mmol}_{\mathrm{c}} \mathrm{dm}^{-3}$; CTC a pH 7, $35 \mathrm{mmol}_{\mathrm{c}} \mathrm{dm}^{-3}$; e saturação por bases (V), 21\%. A granulometria do subsolo, determinada conforme Camargo et al. (1986), foi de 400, 60 e $540 \mathrm{~g} \mathrm{~kg}^{-1}$ de argila, silte e areia, respectivamente.
Os tratamentos corresponderam a quatro doses de esterco e quatro doses de calcário (Tabela 1), combinadas em esquema fatorial $4 \times 4$. O delineamento experimental foi o inteiramente ao acaso com quatro repetições e cada parcela foi composta por cinco mudas, o que totalizou 320 plantas. O substrato foi formado pela mistura de oito partes de subsolo e duas partes de areia grossa, com o acréscimo de doses de esterco correspondentes a 0, 2, 4 e 6 partes (v:v:v). As doses de calcário foram calculadas com base no volume de subsolo de cada tratamento, para elevar o índice de saturação por bases a cerca de 20 (inicial), 35, 50 e $65 \%$, e foi usado calcário dolomítico com PRNT de $91 \%$. O esterco curtido de gado de leite foi secado ao ar, homogeneizado e caracterizado quimicamente $\mathrm{e}$ apresentou: umidade 18\%; $\mathrm{pH}$ em $\mathrm{CaCl}_{2}, 6,1 \mathrm{e}$, na amostra seca, C, $322 \mathrm{~g} \mathrm{~kg}^{-1} ; \mathrm{N}, 10 \mathrm{~g} \mathrm{~kg}^{-1} ; \mathrm{P}, 2,1 \mathrm{~g} \mathrm{~kg}^{-1}$; Ca, 2,0 $\mathrm{g} \mathrm{kg}^{-1} ; \mathrm{Mg}, 1,4 \mathrm{~g} \mathrm{~kg}^{-1} ; \mathrm{K}, 6,8 \mathrm{~g} \mathrm{~kg}^{-1} ; \mathrm{S}, 0,9 \mathrm{~g} \mathrm{~kg}^{-1}$; $\mathrm{Cu}, 26 \mathrm{mg} \mathrm{kg}^{-1} ; \mathrm{Mn}, 136 \mathrm{mg} \mathrm{kg}^{-1} ; \mathrm{Zn}, 65 \mathrm{mg} \mathrm{kg}^{-1}$.

A mistura de subsolo, areia, esterco e calcário foi realizada a seco, de acordo com os tratamentos (Tabela 1). Em todos os tratamentos, até mesmo no testemunha, foram aplicados $150 \mathrm{mg} \mathrm{dm}^{-3} \mathrm{de} \mathrm{P}$, na forma de superfosfato triplo, calculados com base no volume de substrato.

Massa equivalente a $0,8 \mathrm{dm}^{3}$ de substrato foi transferida, com auxílio de um funil, para saquinhos de polietileno de $15 \times 30 \mathrm{~cm}$, providos de furos na metade inferior. Cada conjunto de cinco saquinhos de polietileno constituiu uma parcela e, simultaneamente à condução das plantas, foi mantido um saquinho de substrato sem planta, em incubação, para avaliação da fertilidade no final do experimento.

Tabela1. Proporções e quantidades de subsolo, areia e esterco bovino utilizadas na composição do substrato.

\begin{tabular}{lccccccc}
\hline Tratamento $^{(1)}$ & \multicolumn{3}{c}{ Proporções em volume } & & \multicolumn{3}{c}{ Componentes do substrato $\left(\mathrm{kg} \mathrm{m}^{-3}\right)$} \\
\cline { 2 - 4 } \cline { 6 - 7 } & Subsolo & Areia & Esterco & & Subsolo & Areia & Esterco \\
\hline E0C0 & 8 & 2 & 0 & 1.040 & 310 & 0 \\
E0C1 & 8 & 2 & 0 & 1.040 & 310 & 0 \\
E0C2 & 8 & 2 & 0 & 1.040 & 310 & 0 \\
E0C3 & 8 & 2 & 0 & 1.040 & 310 & 0 \\
E1C0 & 8 & 2 & 2 & 866 & 258 & 101 \\
E1C1 & 8 & 2 & 2 & 866 & 258 & 101 \\
E1C2 & 8 & 2 & 2 & 866 & 258 & 101 \\
E1C3 & 8 & 2 & 2 & 866 & 258 & 101 \\
E2C0 & 8 & 2 & 4 & 743 & 221 & 175 \\
E2C1 & 8 & 2 & 4 & 743 & 221 & 175 \\
E2C2 & 8 & 2 & 4 & 743 & 221 & 175 \\
E2C3 & 8 & 2 & 4 & 743 & 221 & 175 \\
E3C0 & 8 & 2 & 6 & 650 & 194 & 229 \\
E3C1 & 8 & 2 & 6 & 650 & 194 & 229 \\
E3C2 & 8 & 2 & 6 & 650 & 194 & 229 \\
E3C3 & 8 & 2 & 6 & 650 & 194 & 229 \\
\hline
\end{tabular}

${ }^{(1)} \mathrm{E}$ : esterco; C: calcário. 
Em 23 de maio de 2006 foi realizado o transplantio das mudas de guanandi, uma por saquinho. As mudas, fornecidas pela Vasconcelos Florestal, localizada no Município de Monte Alto, SP, tinham, em média, três meses, e haviam sido produzidas em canteiro de areia. $\mathrm{O}$ umedecimento do substrato foi feito com água deionizada, saquinho por saquinho, em quantidade para atingir $90 \%$ da capacidade de retenção de água. $\mathrm{Na}$ primeira semana, a umidade do substrato foi mantida a 90\% da capacidade de retenção de água para melhor pegamento das mudas e, após esse período, a $70 \%$.

No dia do transplantio e aos 60 e 120 dias após, foi determinada a altura das plantas, obtida entre a superfície do substrato e a inserção do último par de folhas do ápice, com régua graduada de precisão de $1 \mathrm{~mm}$. Aos 120 dias foram determinados também o número de folhas; o diâmetro do colo, medido a $2 \mathrm{~cm}$ do substrato, com auxílio de um paquímetro digital modelo CD-6, com precisão de $0,01 \mathrm{~mm}$; e a área foliar, pelo sistema $\Delta \mathrm{T}$ Devices, em folhas de duas mudas, escolhidas ao acaso, de cada repetição, das quais se obteve a matéria seca.

$\mathrm{Na}$ colheita do experimento, aos 120 dias, as plantas foram separadas em raízes, folhas e caule, lavadas em água corrente, em solução de detergente neutro $\left(1 \mathrm{~mL} \mathrm{~L}^{-1}\right)$ e em água deionizada, por três vezes. Em seguida, as plantas foram secadas em estufa com circulação forçada de ar, a aproximadamente $65^{\circ} \mathrm{C}$, até massa constante para obtenção da produção de matéria seca. Conhecida a matéria seca total de folhas, foi calculada a área foliar total, com o emprego da relação entre área foliar das duas plantas avaliadas e a respectiva massa.

O substrato do saquinho extra de cada repetição foi secado, passado em peneira de $2 \mathrm{~mm}$ e homogeneizado para avaliação dos valores de $\mathrm{pH}$ e de condutividade elétrica (relação 1:5), e os teores de matéria orgânica (MO), P, K, Ca e Mg, de acordo com os métodos descritos em Raij et al. (2001).

Os dados obtidos foram submetidos à análise de variância pelo teste $\mathrm{F}$ e os efeitos das doses de cada fator em estudo foram avaliados por meio de análises de regressão polinomial.

\section{Resultados e Discussão}

Em todos os atributos de fertilidade do substrato, foi observado efeito do esterco, mas a calagem só aumentou o pH e os teores de cálcio (Tabela 2). Quanto ao magnésio o efeito da calagem não foi significativo, apesar de ter sido utilizado calcário dolomítico (13\% de $\mathrm{MgO})$ e da variação de $2(\mathrm{C} 0)$ a $6 \mathrm{mmol}_{\mathrm{c}} \mathrm{dm}^{-3}(\mathrm{C} 3)$ nos tratamentos sem esterco (Tabela 2). A aplicação do

Tabela 2. Atributos químicos do substrato depois de 120 dias de incubação.

\begin{tabular}{|c|c|c|c|c|c|c|c|c|}
\hline Esterco & Calcário & P resina & $\mathrm{MO}$ & $\mathrm{pH}\left(\mathrm{CaCl}_{2}\right)$ & $\mathrm{K}^{+}$ & $\mathrm{Ca}^{2+}$ & $\mathrm{Mg}^{2+}$ & $\mathrm{CE}$ \\
\hline$\left(\mathrm{kg} \mathrm{m}^{-3}\right)$ & $\left(\mathrm{g} \mathrm{m}^{-3}\right)$ & $\left(\mathrm{mg} \mathrm{dm}^{-3}\right)$ & $\left(\mathrm{g} \mathrm{dm}^{-3}\right)$ & & \multicolumn{3}{|c|}{-------- $\left(\mathrm{mmol}_{\mathrm{c}} \mathrm{dm}^{-3}\right)$-------- } & $\left(\mu \mathrm{S} \mathrm{cm}^{-1}\right)$ \\
\hline 0 & 0 & 84 & 8 & 4,9 & 0,5 & 7 & 2 & 52 \\
\hline 0 & 228 & 82 & 8 & 5,2 & 0,4 & 9 & 4 & 53 \\
\hline 0 & 557 & 81 & 7 & 5,5 & 0,4 & 10 & 4 & 51 \\
\hline 0 & 865 & 90 & 8 & 5,8 & 0,4 & 11 & 6 & 46 \\
\hline 101 & 0 & 221 & 26 & 5,4 & 15,3 & 15 & 12 & 339 \\
\hline 101 & 228 & 219 & 26 & 5,5 & 17,3 & 18 & 14 & 526 \\
\hline 101 & 557 & 220 & 29 & 5,6 & 16,1 & 17 & 13 & 411 \\
\hline 101 & 865 & 226 & 26 & 5,7 & 16,1 & 18 & 14 & 423 \\
\hline 175 & 0 & 341 & 41 & 5,7 & 25,1 & 24 & 20 & 628 \\
\hline 175 & 228 & 339 & 43 & 5,6 & 32,3 & 30 & 25 & 950 \\
\hline 175 & 557 & 365 & 44 & 5,8 & 32,5 & 29 & 25 & 774 \\
\hline 175 & 865 & 433 & 47 & 6,1 & 37,0 & 32 & 27 & 705 \\
\hline 229 & 0 & 523 & 62 & 6,0 & 43,3 & 34 & 32 & 1.030 \\
\hline 229 & 228 & 481 & 59 & 6,1 & 36,8 & 32 & 29 & 737 \\
\hline 229 & 557 & 481 & 59 & 6,2 & 46,5 & 34 & 34 & 851 \\
\hline \multirow[t]{2}{*}{229} & 865 & 444 & 52 & 6,2 & 34,5 & 31 & 29 & 926 \\
\hline & & \multicolumn{7}{|c|}{ Teste F } \\
\hline Esterco (E) & & $357,32 * *$ & $490,47 * *$ & $64,41 * *$ & $159,12 *$ & $242,2 * *$ & $184,9 * *$ & $24,4 *$ \\
\hline Calcário (C) & & $0,7^{\mathrm{ns}}$ & $0,39^{\mathrm{ns}}$ & $22,98 * *$ & $0,78^{\mathrm{ns}}$ & $3,89 *$ & $1,97^{\mathrm{ns}}$ & $0,1^{\mathrm{ns}}$ \\
\hline Interação E x C & & $2,72 *$ & $2,18^{*}$ & $2,94 * *$ & $2,14^{*}$ & $2,14^{*}$ & $1,22^{\mathrm{ns}}$ & $0,55^{\mathrm{ns}}$ \\
\hline $\mathrm{CV}(\%)$ & & 12,7 & 11,5 & 3,0 & 25,2 & 12,6 & 19,6 & 57,2 \\
\hline
\end{tabular}

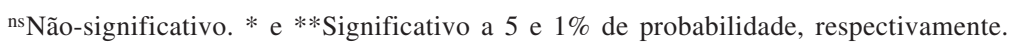


esterco afetou todas as variáveis e, para $\mathrm{pH}, \mathrm{MO}, \mathrm{P}, \mathrm{K}$ e Ca, houve interação entre esterco e calcário (Tabela 2), com obtenção de valores maiores com o aumento do $\mathrm{pH}$ em uma mesma dose de esterco, o que indica que a mineralização do esterco foi estimulada pelo aumento do $\mathrm{pH}$.

$\mathrm{O}$ valor do $\mathrm{pH}$ aumentou linearmente com a aplicação de esterco em todas as doses de calcário (Tabela 3 ). Com a calagem, isoladamente, o $\mathrm{pH}$ aumentou de 4,9 para 5,8, enquanto a aplicação isolada do esterco resultou em aumento nos valores de $\mathrm{pH}$ de 4,9 para 6,0 (Tabela 2). A aplicação de adubos orgânicos pode levar a aumento do valor de $\mathrm{pH}$, como constatado por Yagi et al. (2003) com o uso de esterco e de vermicomposto de esterco bovino, e as explicações prováveis para esse efeito podem ser encontradas em Mantovani et al. (2005).

Em relação ao teor médio de MO no tratamento sem esterco $\left(7,75 \mathrm{~g} \mathrm{dm}^{-3}\right)$ houve aumento de $19 \mathrm{~g} \mathrm{dm}^{-3} \mathrm{de}$ MO no tratamento que recebeu $101 \mathrm{~kg} \mathrm{~m}^{-3}$ de esterco. Nos demais tratamentos, que receberam 175 e $229 \mathrm{~kg} \mathrm{~m}^{-3}$ de esterco, os aumentos médios foram de 36 e $50 \mathrm{~g} \mathrm{dm}^{-3}$, respectivamente (Tabela 2 ). Com estes aumentos, considerando o teor de C orgânico do esterco $\left(322 \mathrm{~g} \mathrm{~kg}^{-1}\right)$, as doses aplicadas, e o teor final de MO no substrato em cada tratamento, tem-se que 34 (E1), 37 (E2) e 39\% (E3) do $\mathrm{C}$ orgânico adicionado foi incorporado à MO estável do substrato, após quatro meses de incubação. No substrato que não recebeu calcário, a variação no teor de MO de acordo com as doses de esterco foi quadrática e, nos demais tratamentos $(\mathrm{C} 1, \mathrm{C} 2$ e $\mathrm{C} 3)$, linear (Tabela 3). Yagi et al. (2003) também relataram aumento do teor de MO do solo com aplicação de esterco e de vermicomposto de esterco bovino, em que $70 \mathrm{t} \mathrm{ha}^{-1}$ de esterco seriam necessárias para produzir a mesma quantidade de $\mathrm{MO}$ gerada por $39 \mathrm{tha}^{-1}$ de vermicomposto.

$\mathrm{O}$ teor de $\mathrm{P}$ no substrato aumentou em função das doses de esterco, segundo um modelo quadrático para as doses de calcário correspondentes a $\mathrm{C} 0(\mathrm{~V}, 20 \%)$, $\mathrm{C} 1(\mathrm{~V}, 35 \%)$ e $\mathrm{C} 2(\mathrm{~V}, 50 \%)$, ao passo que para $\mathrm{C} 3(\mathrm{~V}$, $65 \%$ ) foi observado aumento linear (Tabela 3). O teor de $\mathrm{P}$ no substrato atingiu $523 \mathrm{mg} \mathrm{dm}^{-3} \mathrm{com}$ a aplicação do esterco, no tratamento sem calagem, o que representa variação de 6,2 vezes (Tabela 2). Aumentos nos teores de $\mathrm{P}$ foram também verificados por Alves et al. (1999), com aplicação de composto de lixo urbano, e por Yagi et al. (2003), com a aplicação de esterco e de vermicomposto de esterco.

Quanto ao teor de K no substrato, foi observado efeito quadrático de acordo com as doses de esterco, no tratamento que não recebeu calcário $(\mathrm{C} 0)$, e aumento linear nos tratamentos com calagem para $\mathrm{C} 1, \mathrm{C} 2$ e C3 (Tabela 3). No tratamento sem calagem, a variação nos

Tabela 3. Regressões para os atributos químicos do substrato (y) em função das doses de esterco (x) em cada condição de calagem para atingir diferentes valores de saturação por bases (V).

\begin{tabular}{lccc}
\hline Atributos & $\mathrm{V}(\%)$ & Equação & $\mathrm{R}^{2}$ \\
\hline $\mathrm{pH}$ & 20 & $\mathrm{y}=0,004 \mathrm{x}+4,880$ & $0,991^{* *}$ \\
& 35 & $\mathrm{y}=0,003 \mathrm{x}+5,105$ & $0,933^{* *}$ \\
& 50 & $\mathrm{y}=0,003 \mathrm{x}+5,383$ & $0,665^{* *}$ \\
\hline $\mathrm{MO}\left(\mathrm{g} \mathrm{dm}^{-3}\right)$ & 65 & $\mathrm{y}=0,002 \mathrm{x}+5,667$ & $0,995^{* *}$ \\
& 20 & $\mathrm{y}=0,0005 \mathrm{x}^{2}+0,098 \mathrm{x}+8,388$ & $0,985^{* *}$ \\
& 35 & $\mathrm{y}=0,219 \mathrm{x}+6,412$ & $0,996^{* *}$ \\
\hline $\mathrm{P}\left(\mathrm{mg} \mathrm{dm}^{-3}\right)$ & 50 & $\mathrm{y}=0,222 \mathrm{x}+6,522$ & $0,979^{* *}$ \\
& 65 & $\mathrm{y}=0,206 \mathrm{x}+7,173$ & $0,993^{* *}$ \\
& 20 & $\mathrm{y}=0,0054 \mathrm{x}^{2}+0,608 \mathrm{x}+87,461$ & $0,998^{*}$ \\
$\mathrm{~K}\left(\mathrm{mmol}_{\mathrm{c}} \mathrm{dm}^{-3}\right)$ & 35 & $\mathrm{y}=0,0036 \mathrm{x}^{2}+0,888 \mathrm{x}+83,969$ & $0,999^{* *}$ \\
& 50 & $\mathrm{y}=1,684 \mathrm{x}+85,440$ & $0,949^{* *}$ \\
\hline $\mathrm{Ca}\left(\mathrm{mmol}_{\mathrm{c}} \mathrm{dm}^{-3}\right)$ & 65 & $\mathrm{y}=0,0005 \mathrm{x}^{2}+0,074 \mathrm{x}+0,929$ & $0,986^{*}$ \\
& 20 & $\mathrm{y}=0,165 \mathrm{x}+0,837$ & $0,987^{* *}$ \\
& 35 & $\mathrm{y}=0,200 \mathrm{x}-1,407$ & $0,986^{* *}$ \\
& 50 & $\mathrm{y}=0,166 \mathrm{x}+1,065$ & $0,918^{* *}$ \\
\hline
\end{tabular}

* e **Significativo a 5 e $1 \%$ de probabilidade, respectivamente. 
teores de K com a aplicação do esterco foi de 0,5 a $43,3 \mathrm{mmol}_{\mathrm{c}} \mathrm{dm}^{-3}$, o que demonstra alto potencial do adubo em fornecer este nutriente e, em sistemas com irrigação controlada, pode ser o fator limitante na definição da dose de esterco, uma vez que há aumento simultâneo da concentração salina e da condutividade elétrica que, no caso, aumentou segundo a equação $\mathrm{y}=-0,00242 \mathrm{x}^{2}+4,31146 \mathrm{x}+43,52173\left(\mathrm{R}^{2}=0,993 * *\right)$.

A aplicação do esterco resultou em efeito quadrático nos teores de $\mathrm{Ca}$ do substrato nos tratamentos com calagem para V $20(\mathrm{C} 0)$ e $50 \%(\mathrm{C} 2)$, e em aumento linear para V 35 (C1) e 65\% (C3) (Tabela 3). O Ca atingiu teor de $34 \mathrm{mmol}_{\mathrm{c}} \mathrm{dm}^{-3} \mathrm{com}$ a aplicação da maior dose de esterco, e foi 4,9 vezes superior ao do tratamento testemunha (Tabela 2). Quanto ao Mg, foi observado aumento linear de acordo com as doses de esterco $(y=0,119 x+2,933)$, e a variação foi de 2 para
$34 \mathrm{mmol}_{\mathrm{c}} \mathrm{dm}^{-3}$ (Tabela 2). Alves et al. (1999) e Yagi et al. (2003) também verificaram aumento dos teores de $\mathrm{K}, \mathrm{Ca}$ e $\mathrm{Mg}$ com a aplicação de composto de lixo urbano e de esterco e vermicomposto de esterco bovino, respectivamente.

A aplicação da menor dose de esterco bovino foi suficiente para elevar o $\mathrm{pH}$ e os teores de $\mathrm{P}, \mathrm{K}, \mathrm{Ca}$ e $\mathrm{Mg}$ a valores muito altos, conforme as classes de fertilidade para esses atributos em uso no Estado de São Paulo (Raij et al., 1996). No entanto, na produção de mudas de guanandi, a dose de esterco que vem sendo usada é a que corresponde à maior dose estudada.

Em todas as variáveis de crescimento do guanandi, houve efeito das doses de esterco, mas não do calcário, e a interação entre ambos não foi significativa (Tabela 4). Gomes et al. (2004) não observaram resposta em altura do angico-branco (Anadenanthera colubrina (Vell.)

Tabela 4. Variáveis de crescimento das mudas de guanandi em função das doses de esterco associadas às doses de calcário ${ }^{(1)}$.

\begin{tabular}{|c|c|c|c|c|c|c|c|c|c|c|c|c|}
\hline Esterco & Calcário & $\mathrm{A} 1$ & $\mathrm{~A} 2$ & $\mathrm{D}$ & $\mathrm{A} 2 / \mathrm{D}$ & NF & MST & MSC & MSR & MSF & MSPA & $\mathrm{AF}$ \\
\hline$\left(\mathrm{kgm}^{-3}\right)$ & $\left(\mathrm{g} \mathrm{m}^{-3}\right)$ & \multicolumn{2}{|c|}{$----(\mathrm{cm})----$} & $(\mathrm{mm})$ & & & \multicolumn{5}{|c|}{ 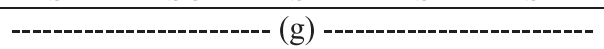 } & $\left(\mathrm{cm}^{2}\right)$ \\
\hline 0 & 0 & 20,0 & 27,9 & 3,84 & 7,3 & 12,8 & 16,69 & 3,35 & 7,19 & 6,67 & 10,02 & 352,5 \\
\hline 0 & 228 & 21,5 & 29,9 & 3,96 & 7,6 & 12,0 & 18,90 & 3,85 & 7,30 & 7,53 & 11,38 & 419,7 \\
\hline 0 & 557 & 21,1 & 28,5 & 3,90 & 7,3 & 13,0 & 16,23 & 3,46 & 6,36 & 6,39 & 9,84 & 333,6 \\
\hline 0 & 865 & 22,9 & 30,1 & 4,02 & 7,5 & 12,0 & 16,70 & 3,75 & 7,12 & 6,47 & 10,23 & 371,3 \\
\hline Médias & & 21,2 & 29,1 & 3,93 & 7,4 & 12,5 & 17,13 & 3,60 & 6,99 & 6,77 & 10,37 & 369,3 \\
\hline 101 & 0 & 21,3 & 32,5 & 3,81 & 8,5 & 12,3 & 15,18 & 3,31 & 5,09 & 5,94 & 9,25 & 481,5 \\
\hline 101 & 228 & 20,8 & 29,0 & 3,77 & 7,7 & 11,5 & 13,42 & 3,16 & 4,93 & 5,13 & 8,29 & 427,2 \\
\hline 101 & 557 & 21,5 & 31,2 & 3,99 & 7,8 & 12,8 & 15,34 & 3,53 & 5,57 & 5,90 & 9,44 & 483,3 \\
\hline 101 & 865 & 20,6 & 28,7 & 3,80 & 7,5 & 11,5 & 13,05 & 3,19 & 4,69 & 4,93 & 8,12 & 428,2 \\
\hline Médias & & 21,1 & 30,4 & 3,84 & 7,9 & 12,0 & 14,25 & 3,30 & 5,07 & 5,48 & 8,78 & 455,1 \\
\hline 175 & 0 & 21,4 & 28,8 & 3,91 & 7,4 & 11,8 & 11,82 & 3,15 & 4,50 & 4,34 & 7,49 & 380,4 \\
\hline 175 & 228 & 20,6 & 27,8 & 3,76 & 7,4 & 10,8 & 11,06 & 2,94 & 4,97 & 4,06 & 7,00 & 338,7 \\
\hline 175 & 557 & 19,7 & 26,4 & 3,65 & 7,2 & 10,5 & 9,77 & 2,59 & 4,28 & 3,59 & 6,18 & 309,0 \\
\hline 175 & 865 & 19,8 & 24,4 & 3,64 & 6,7 & 10,3 & 9,68 & 2,49 & 4,41 & 3,59 & 6,08 & 340,0 \\
\hline Médias & & 20,4 & 26,9 & 3,74 & 7,2 & 10,9 & 10,58 & 2,79 & 4,54 & 3,90 & 6,69 & 342,0 \\
\hline 229 & 0 & 18,9 & 23,5 & 3,49 & 6,7 & 10,3 & 8,40 & 2,21 & 4,21 & 3,10 & 5,31 & 262,1 \\
\hline 229 & 228 & 16,9 & 21,1 & 3,60 & 5,9 & 9,3 & 6,11 & 1,63 & 3,52 & 2,24 & 3,87 & 221,2 \\
\hline 229 & 557 & 20,4 & 22,8 & 3,41 & 6,7 & 9,5 & 7,28 & 1,85 & 3,74 & 2,66 & 4,62 & 229,3 \\
\hline 229 & 865 & 19,2 & 22,8 & 3,53 & 6,5 & 9,8 & 7,29 & 1,86 & 4,43 & 2,72 & 4,57 & 250,1 \\
\hline \multicolumn{2}{|l|}{ Médias } & 18,9 & 22,6 & 3,51 & 6,5 & 9,7 & 7,27 & 1,89 & 3,98 & 2,68 & 4,59 & 240,7 \\
\hline & & \multicolumn{11}{|c|}{ Teste $\mathrm{F}$} \\
\hline \multicolumn{2}{|c|}{ Esterco (E) } & $6,5^{* *}$ & $35,7 * *$ & $10,1 * *$ & $23,9 * *$ & $22,3 * *$ & $78,9 * *$ & $38,0^{* *}$ & $42,2 * *$ & $89,3 * *$ & $71,4 * *$ & $38,4 * *$ \\
\hline \multicolumn{2}{|c|}{ Calcário (C) } & $0,6^{\mathrm{ns}}$ & $1,5^{\mathrm{ns}}$ & $0,7^{\mathrm{ns}}$ & $2,1^{\mathrm{ns}}$ & $2,7^{\mathrm{ns}}$ & $1,3^{\mathrm{ns}}$ & $0,4^{\mathrm{ns}}$ & $0,3^{\mathrm{ns}}$ & $1,6^{\mathrm{ns}}$ & $1,1^{\mathrm{ns}}$ & $0,8^{\mathrm{ns}}$ \\
\hline \multicolumn{2}{|c|}{ Interação E x C } & $1,7^{\mathrm{ns}}$ & $1,8^{\text {ns }}$ & $0,9^{\mathrm{ns}}$ & $1,7^{\mathrm{ns}}$ & $0,6^{\mathrm{ns}}$ & $1,3^{\mathrm{ns}}$ & $1,2^{\text {ns }}$ & $1,1^{\mathrm{ns}}$ & $1,3^{\mathrm{ns}}$ & $1,2^{\text {ns }}$ & $1,2^{\mathrm{ns}}$ \\
\hline \multicolumn{2}{|c|}{ CV (\%) } & 8,6 & 8,5 & 6,1 & 6,8 & 9,3 & 15,7 & 16,8 & 15,7 & 16,1 & 15,6 & 16,2 \\
\hline
\end{tabular}

(1)A1: altura aos 60 dias; A2: altura aos 120 dias; D: diâmetro; A2/D: relação altura aos 120 dias/diâmetro; NF: número de folhas; MST: matéria seca total; MSC: matéria seca do caule; MSR: matéria seca da raiz; MSF: matéria seca das folhas; MSPA: matéria seca da parte aérea; AF: área foliar; equações relativas aos efeitos das doses de esterco em: $\mathrm{A} 1, \mathrm{y}=-0,0101 \mathrm{x}+21,696, \mathrm{R}^{2}=0,8077 * * \mathrm{~A} 2, \mathrm{y}=-0,0003 \mathrm{x}^{2}+0,0426 \mathrm{x}+29,126$, $\mathrm{R}^{2}=0,9988 * * ; \mathrm{D}, \mathrm{y}=-9 \mathrm{E}-06 \mathrm{x}^{2}+0,0004 \mathrm{x}+3,9240, \mathrm{R}^{2}=0,9791 * ; \mathrm{A} 2 / \mathrm{D}, \mathrm{y}=-7 \mathrm{E}-05 \mathrm{x}^{2}+0,0102 \mathrm{x}+7,4396, \mathrm{R}^{2}=0,9883 * * ; \mathrm{NF}, \mathrm{y}=-6 \mathrm{E}-05 \mathrm{x}^{2}$ $+0,0012 \mathrm{x}+12,458, \mathrm{R}^{2}=0,9991 * ; \mathrm{AF}, \mathrm{y}=-0,0101 \mathrm{x}^{2}+1,701 \mathrm{x}+372,53, \mathrm{R}^{2}=0,9733 * * ; \mathrm{MST}, \mathrm{y}=-0,0001 \mathrm{x}^{2}-0,0177 \mathrm{x}+17,138, \mathrm{R}^{2}=0,9999 * ;$ MSF, $y=-0,0179 x+6,959, R^{2}=0,9813 * * ; M S C, y=-4 E-05 x^{2}+0,0019 x+3,5857, R^{2}=0,9902 * * ; M S P A, y=-7 E-05 x^{2}-0,008 x+10,364$, $\mathrm{R}^{2}=1 * ;$ MSR $\mathrm{y}=4 \mathrm{E}-05 \mathrm{x}^{2}-0,0214 \mathrm{x}+6,974, \mathrm{R}^{2}=0,9910 *$. ns Não-significativo. * e **Significativo a 5 e $1 \%$ de probabilidade, respectivamente. 
Brenan) à calagem, o que foi relacionado aos teores de $\mathrm{Ca}\left(9 \mathrm{mmol}_{\mathrm{c}} \mathrm{dm}^{-3}\right)$ e de $\mathrm{Mg}\left(4 \mathrm{mmol}_{\mathrm{c}} \mathrm{dm}^{-3}\right)$ do solo, considerados suficientes para suprir as necessidades das plantas nessa fase. O solo que foi utilizado para o guanandi apresentava teores de $\mathrm{Ca}$ e $\mathrm{Mg}$ menores, mas mesmo assim foram suficientes.

No transplantio, a altura média das mudas selecionadas foi de $14,4 \mathrm{~cm}$. Nas duas medidas feitas posteriormente, aos 60 e 120 dias após o transplantio, as alturas variaram, respectivamente, de 16,9 a $22,9 \mathrm{~cm}$ e de 21,1 a $32,5 \mathrm{~cm}$ (Tabela 4). Na avaliação realizada aos 60 dias observouse que, à medida que aumentou a dose de esterco, houve menor crescimento das plantas, o que resultou em efeito linear negativo (Tabela 4). O efeito das doses de esterco na altura da planta, medida aos 120 dias do transplantio, foi quadrático, e a altura máxima foi correspondente à dose de $71 \mathrm{~kg} \mathrm{~m}^{-3}$ de esterco (Tabela 4). Aos 60 dias, a diferença na altura das plantas entre o tratamento que não recebeu esterco e o que recebeu a maior dose de esterco foi de $11 \%$, e aos 120 dias, de 22,5\%. De acordo com Gonçalves et al. (2000), para mudas de plantas florestais de boa qualidade, os valores adequados para altura estão entre 20 e $35 \mathrm{~cm}$. Nas avaliações feitas aos 60 dias e aos 120 dias, 55 e $88 \%$ das mudas, respectivamente, apresentaram valores de altura dentro do intervalo de 20 a $35 \mathrm{~cm}$. A maior porcentagem de mudas que atendeu ao padrão de qualidade mencionado foi obtida no tratamento testemunha, tanto aos 60 quanto aos 120 dias do transplantio. A adubação orgânica normalmente resulta em efeitos benéficos na formação de mudas de espécies florestais, como os relatados para oiti (Licania tomentosa Benth.) adubado com composto de lixo urbano (Alves \& Passoni, 1997) e angelim (Andira fraxinifolia Benth.) adubado com esterco bovino (Carvalho Filho et al., 2004). No entanto, quanto ao guanandi este efeito não foi observado com as doses estudadas.

No tratamento que não recebeu esterco e calcário, o diâmetro do colo das mudas, medido aos 120 dias, foi $10,7 \%$ maior do que no tratamento que recebeu a maior dose de esterco $\left(229 \mathrm{~kg} \mathrm{~m}^{-3}\right)$. O efeito das doses de esterco no diâmetro do colo foi quadrático (Tabela 4) e, de acordo com a equação obtida, $22 \mathrm{~kg} \mathrm{~m}^{-3}$ de esterco são suficientes para alcançar o diâmetro máximo. $\mathrm{Na}$ produção de mudas de Acacia mearnsii Wild (acácianegra), Caldeira et al. (2000) observaram que houve diminuição no diâmetro do colo nas doses de vermicomposto superiores a $112 \mathrm{~cm}^{3}$ por $280 \mathrm{~cm}^{3} \mathrm{de}$ substrato. Vogel et al. (2001) verificaram efeito positivo para o diâmetro do colo com uso de até $40 \%$ de vermicomposto na composição do substrato para Hovenia dulcis (Thumberg) e Souza et al. (2001) observaram que os melhores resultados para diâmetro de caule de mudas de cagaita (Eugenia dysenterica DC.) foram obtidos no substrato solo + terra vegetal + vermiculita, sem ou com adubo químico, nas proporções 1:1:2.

De acordo com Daniel et al. (1997), o diâmetro do colo é avaliado para indicar a capacidade de sobrevivência da muda no campo e uma muda de Acacia mangium de qualidade deve apresentar diâmetro maior do que $2 \mathrm{~mm}$. Por sua vez, Gonçalves et al. (2000) consideram que o diâmetro do colo adequado a mudas de espécies florestais de qualidade está entre 5 e $10 \mathrm{~mm}$. O maior valor médio aos 120 dias para diâmetro do colo das mudas de guanandi foi $4,02 \mathrm{~mm}$. Portanto, todas as mudas apresentaram diâmetro superior ao recomendado por Daniel et al. (1997) para A. mangium e $100 \%$ ficaram com diâmetro inferior a $5 \mathrm{~mm}$, mínimo sugerido por Gonçaves et al. (2000). As variações no diâmetro do colo ocorrem de acordo com a espécie e ainda não há relatos quanto ao guanandi, mas como aos 120 dias as mudas de guanandi haviam atingido o padrão de comercialização adotado por viveiristas, o intervalo de valores de diâmetro do colo sugerido por Gonçalves et al. (2000) parece não ser aplicável para esta espécie.

A altura média das plantas variou de $30,35 \mathrm{~cm}$, no tratamento que recebeu $101 \mathrm{~kg} \mathrm{~m}^{-3}$ de esterco (E1), a $22,55 \mathrm{~cm}$, quando foram aplicados $229 \mathrm{~kg} \mathrm{~m}^{-3}$ (E3). Por sua vez, o diâmetro do colo variou de 3,93 a $3,51 \mathrm{~cm}$, médias alcançadas para os tratamentos E0 e E3, respectivamente. Deste modo, das duas variáveis, a altura foi a medida mais sensível às alterações provocadas pela aplicação do esterco e a principal responsável pela variação na relação altura/diâmetro. O efeito das doses de esterco na relação altura/diâmetro do colo foi quadrático (Tabela 4) e a diminuição da relação com a aplicação de $229 \mathrm{~kg} \mathrm{~m}^{-3}$ de esterco foi de $18 \%$ em relação ao tratamento que recebeu $101 \mathrm{~kg} \mathrm{~m}^{-3}$.

De acordo com Sturion \& Antunes (2000), a relação altura/diâmetro do colo é utilizada para avaliar a qualidade das mudas florestais, pois, além de refletir o acúmulo de reservas, assegura maior resistência e melhor fixação no solo. Mudas com diâmetro do colo menor apresentam dificuldades para se manter eretas após o plantio e o tombamento pode resultar em morte ou deformações, que comprometem o valor silvicultural da planta. Mudas com diâmetro do colo pequeno e alturas elevadas são consideradas de qualidade inferior em relação às menores 
em altura e com maior diâmetro do colo. Essa relação é reconhecida como um dos melhores, se não o melhor, indicadores do padrão de qualidade de mudas (Moreira \& Moreira, 1996), sendo, em geral, o mais indicado para determinar a capacidade de sobrevivência no campo (Daniel et al., 1997). Quanto ao guanandi, as mudas do tratamento-testemunha foram as que apresentaram maior diâmetro do colo e também maior altura, ou seja, foram as mudas com melhor qualidade para serem levadas ao campo.

Foi observado efeito quadrático das doses de esterco no número de folhas (Tabela 4). O maior valor estimado (12 folhas) foi obtido no tratamento que não recebeu esterco. Nas mudas produzidas no substrato sem adição de esterco, o número de folhas foi semelhante ao do tratamento que recebeu $101 \mathrm{~kg} \mathrm{~m}^{-3}$ de esterco, e foi $12,9 \%$ e $21,8 \%$ superior aos tratamentos que receberam 175 e $229 \mathrm{~kg} \mathrm{~m}^{-3}$ de esterco, respectivamente. Em média, os tratamentos sem esterco e com a maior dose de esterco apresentaram de 12 a 14 e de 6 a 10 folhas, respectivamente. Carvalho Filho et al. (2002) obtiveram melhores resultados para número de folhas de canafístula (Cassia grandis L.) em substrato composto por solo + esterco (2:1) e solo + areia + esterco (1:2:1) (v:v:v), com 12,4 e 12,0 folhas por planta, respectivamente.

$\mathrm{A}$ área foliar se correlaciona diretamente com a área da superfície fotossintetizante útil e foi observado efeito quadrático das doses de esterco na área foliar das mudas de guanandi (Tabela 4). A maior média foi verificada no tratamento que recebeu $101 \mathrm{~kg} \mathrm{~m}^{-3}$ de esterco, sendo 18,9, 24,8 e $47,1 \%$ superior à dos tratamentos sem esterco e com aplicação de 175 e $229 \mathrm{~kg} \mathrm{~m}^{-3}$ de esterco, respectivamente.

As produções de matéria seca das folhas (MSF), do caule (MSC), da parte aérea (MSPA), das raízes (MSR) e matéria seca total (MST) diminuíram com o aumento das doses de esterco bovino. Com exceção da MSF obteve-se, para todas essas variáveis, efeito quadrático das doses de esterco. A MSF apresentou comportamento linear decrescente para as doses de esterco (Tabela 4). A MSF, a MSC, a MSPA e a MSR do tratamentotestemunha foram 60,4, 47,6, 55,7 e 43,2\% maiores em relação ao tratamento que recebeu a maior dose de esterco e a MST foi 57,6\% maior. Caldeira et al. (2000) observaram que, com o aumento das doses de vermicomposto no substrato, ocorreu aumento da matéria seca total, da parte aérea e das raízes de plantas de Eucalyptus saligna Smith. Essa constatação também foi feita, em estudo semelhante, por Tedesco et al. (1999) para Jacaranda micrantha Chamisso, quando utilizaram vermicomposto. Cunha et al. (2005) observaram que mudas de Tabebuia impetiginosa (Mart. ex D.C.) Standl (ipê-roxo) apresentaram maior produção de matéria seca da parte aérea, quando cultivadas em substrato constituído por subsolo + composto orgânico, e que apenas o subsolo não atendeu às exigências nutricionais da espécie em viveiro.

A diminuição do crescimento, altura, diâmetro, número de folhas, área foliar e matéria seca, das mudas de guanandi provocada pela adição de esterco ao substrato, que contraria observações verificadas em outras espécies florestais, está relacionada ao aumento nos teores de nutrientes e da condutividade elétrica que foram obtidos. A aplicação de $101 \mathrm{~kg} \mathrm{~m}^{-3}$ foi suficiente para elevar os teores de nutrientes e o $\mathrm{pH}$ a valores muito altos de acordo com as classes de fertilidade do solo. Quanto às variáveis MST e MSPA, foram obtidas correlações negativas com os teores de P, K, Ca e Mg (r: -0,96, -0,95, -0,96 e $-0,96$, respectivamente) e com os valores de $\mathrm{pH}$ e CE (r: $-0,84$ e $-0,91$, respectivamente) no substrato. Nas demais variáveis, os resultados das correlações foram semelhantes. As doses de esterco avaliadas estão dentro do intervalo de doses que são utilizadas na composição de substratos para produção de mudas de guanandi em viveiros comerciais, mas como foi realizado controle de irrigação para evitar perdas por lixiviação, a quantidade de sais acumulada foi excessiva para a espécie, o que provocou necrose nas folhas das plantas que foram adubadas com a maior dose de esterco. O esterco é um componente importante do substrato, particularmente por aumentar o teor de matéria orgânica e a CTC, e, por isso, o seu uso não pode ser descartado. A dose precisa ser ajustada, porém.

A resposta de crescimento do guanandi é condizente com a hipótese de que as espécies climácicas mostram crescimento pouco influenciado pelo nível de fertilidade do solo, o que, segundo Siqueira et al. (1995), pode ser indicativo de maior adaptação a solos pouco férteis, ou de rígido ajuste da taxa de crescimento às condições de baixa disponibilidade de nutrientes, o que restringe sua resposta à melhoria nos níveis de fertilidade do solo.

\section{Conclusão}

A calagem e a adubação com esterco bovino não são necessárias para produção de mudas de guanandi. 


\section{Referências}

ALVES, W.L.; MELO, W.J.; FERREIRA, M.E. Efeito do composto de lixo urbano em um solo arenoso e em plantas de sorgo. Revista Brasileira de Ciência do Solo, v.23, p.729-736, 1999.

ALVES, W.L.; PASSONI, A.A. Composto e vermicomposto de lixo urbano na produção de mudas de oiti (Licania tomentosa Benth.) para arborização. Pesquisa Agropecuária Brasileira, v.32, p.5862, 1997.

BALIEIRO, F.C.; OLIVEIRA, I.G.; DIAS, L.E. Formação de mudas de Acacia holosericea e A. auriculiformis: resposta à calagem, fósforo, potássio e enxofre. Revista Árvore, v.25, p.183-191, 2001.

CALDEIRA, M.V.W.; SCHUMACHER, M.V.; TEDESCO, N. Crescimento de mudas de Acacia mearnsii em função de diferentes doses de vermicomposto. Scientia Florestalis, v.57, p.161-170, 2000.

CAMARGO, O.A.; MONIZ, A.C.; JORGE, J.A.; VALADARES, J.M.A.S. Métodos de análise química, mineralógica e física de solos do Instituto Agronômico de Campinas. Campinas: Instituto Agronômico, 1986. 94p. (Boletim técnico, 106).

CARVALHO, P.E.R. Espécies florestais brasileiras: recomendações silviculturais, potencialidades e uso da madeira. Curitiba: Embrapa-CNPF, 1994. 572p.

CARVALHO FILHO, J.L.S.; ARRIGONI-BLANK, M.F.; BLANK, A.F. Produção de mudas de angelim (Andira fraxinifolia Benth.) em diferentes ambientes, recipientes e substratos. Revista Ciência Agronômica, v.35, p.61-67, 2004.

CARVALHO FILHO, J.L.S.; ARRIGONI-BLANK, M.F.; BLANK, A.F.; SANTOS NETO, A.L.; AMÂNCIO, V.F. Produção de mudas de Cassia grandis L. em diferentes ambientes, recipientes e misturas de substratos. Revista Ceres, v.40, p.341-352, 2002.

CASTRO, E.M.; ALVARENGA, A.A.; GOMIDE, M.B.; GEISENHOFF, L.O. Efeito de substrato na produção de mudas de calabura (Muntingia calabura L.). Ciência e Agrotecnologia, v.20, p.366-370, 1996.

CUNHA, A.O.; ANDRADE, L.A.; BRUNO, R.L.A.; SILVA, J.A.L.; SOUZA, V.C. Efeitos de substratos e das dimensões dos recipientes na qualidade das mudas de Tabebuia impetiginosa (Mart. Ex D.C.) Standl. Revista Árvore, v.29, p.507-516, 2005

DANIEL, O.; VITORINO, A.C.T.; ALOISI, A.A.; MAZZOCHIN, L.; TOKURA, A.M.; PINHEIRO, E.R.; SOUZA, E.F. Aplicação de fósforo em mudas de Acacia mangium. Revista Árvore, v.21, p.163$168,1997$.

FERNANDES, L.A.; FURTINI NETO, A.E.; FONSECA, F.C.; VALE, F.R. Crescimento inicial, níveis críticos de fósforo e frações fosfatadas em espécies florestais. Pesquisa Agropecuária Brasileira, v.35, p.1191-1198, 2000.

FURTINI NETO, A.E.; RESENDE, A.V.; VALE, F.R.; FAQUIN, V.; FERNANDES, L.A. Acidez do solo, crescimento e nutrição mineral de algumas espécies arbóreas, na fase de muda. Cerne, v.5, p.1-12, 1999.
GOMES, J.M.; COUTO, L. Produção de mudas de eucalipto. Informe Agropecuário, v.12, p.8-14, 1986.

GOMES, K.C.O.; PAIVA, H.N.; NEVES, J.C.L.; BARROS, N.F.B.; SILVA, S.R. Influência da saturação por bases e do fósforo no crescimento de mudas de angico-branco. Revista Árvore, v.28, p.785792,2004

GONÇALVES, J.L.M.; SANTARELLI, E.G.; MORAES NETO, S.P.; MANARA, M.P. Produção de mudas de espécies nativas: substrato, nutrição, sombreamento e fertilização. In: GONÇALVES, J.L.M.; BENEDETTI, V. (Ed.). Nutrição e fertilização florestal. Piracicaba: Ipef, 2000. p.309-350.

MANTOVANI, J.R.; FERREIRA, M.E.; CRUZ, M.C.P.; BARBOSA, J.C. Alterações nos atributos de fertilidade em solo adubado com composto de lixo urbano. Revista Brasileira de Ciência do Solo, v.29, p.817-824, 2005.

MOREIRA, F.M.S.; MOREIRA, F.W. Característica de germinação de 64 espécies de leguminosas florestais nativas da Amazônia, em condições de viveiro. Acta Amazônica, v.26, p.3-16, 1996.

RAIJ, B. van; ANDRADE, J.C.; CANTARELLA, H.; QUAGGIO, J.A. (Ed.). Análises químicas para avaliação da fertilidade de solos tropicais. Campinas: Instituto Agronômico, 2001. 285p.

RAIJ, B. van; CANTARELLA, H.; QUAGGIO, J.A.; FURLANI, A.M.C. Interpretação de resultados de análise de solo. In: RAIJ, B. van; CANTARELLA, H.; QUAGGIO, J.A.; FURLANI, A.M.C. (Ed.). Recomendações de adubação e calagem para o Estado de São Paulo. Campinas: Instituto Agronômico-Fundação IAC, 1996. p.8-13. (Boletim técnico, 100).

SIQUEIRA, J.O.; CURI, N.; VALE, F.R.; FERREIRA, M.M.; MOREIRA, F.M.S. Aspectos de solos, nutrição vegetal e microbiologia na implantação de matas ciliares. Belo Horizonte: Cemig, 1995. 28p.

SOUZA, E.R.; CARNEIRO, I.F.; NAVES, R.V.; BORGES, J.D.; LEANDRO, W.M.; CHAVES, L.J. Emergência e crescimento de cagaita (Eugenia dysenterica DC.) em função do tipo e do volume de substratos. Pesquisa Agropecuária Tropical, v.31, p.89-95, 2001.

STURION, J.A.; ANTUNES, J.B.M. Produção de mudas de espécies florestais. In: GALVÃO, A.P.M. (Ed.). Reflorestamento de propriedades rurais para fins produtivos e ambientais. Colombo: Embrapa Florestas, 2000. p.125-150.

TEDESCO, N.; CALDEIRA, M.V.W.; SCHUMACHER, M.V. Influência do vermicomposto na produção de mudas de caroba (Jacaranda micrantha Chamisso). Revista Árvore, v.23, p.1-8, 1999.

VOGEL, H.L.M.; SCHUMACHER, M.V.; BARICHELO, L.R.; OLIVEIRA, L.S.; CALDEIRA, M.V.W. Utilização de vermicomposto no crescimento de mudas de Hovenia dulcis Thunberg. Ciência Florestal, v.11, p.1053-1058, 2001.

YAGI, R.; FERREIRA, M.E.; CRUZ, M.C.P.; BARBOSA, J.C. Organic matter fractions and soil fertility under the influence of liming, vermicompost and cattle manure. Scientia Agricola, v.60, p.549-557, 2003 .

Recebido em 28 de novembro de 2006 e aprovado em 4 de maio de 2007 